\title{
Gibt es ein Patentrezept für erfolgreiche IT-Projekte?
}

Alfred Katzenbach

Viele Prozess-IT-Projekte erfüllen nicht die Erwartungen des Managements, der Anwender, oder von beiden. Ein beachtlicher Anteil wird abgebrochen und scheitert damit komplett. Aber gerade der Erfolg dieser Projekte kann für den Erfolg des ganzen Unternehmens entscheidend sein. Wenn man diese Projekte als Organisationsentwicklungsprojekte mit einem klaren Fokus auf die beteiligten und betroffenen Personen betrachtet, steigt die Erfolgswahrscheinlichkeit in großem Maß. Der vorgestellte Fünf-Phasen-Plan zeigt ein Vorgehensmodell, mit dem auch große internationale und komplexe IT-Projekte erfolgreich bearbeitet werden können und wie ein nachhaltiger Erfolg für das Unternehmen sichergestellt werden kann.

Keywords: Projektmanagement, IT-Projekte, Organisationsentwicklung, Einführungsplan

\section{Motivation}

Sehr viele IT-Einführungsprojekte erfüllen nicht die Erwartungen, dauern viel länger oder überziehen das geplante Budget. Nicht wenige scheitern. Lässt sich dies vermeiden? Ich bin der festen Überzeugung: Ja, es gibt einen Weg zu erfolgreichen Projekten - „in time, in budget, in quality“. Der Chaos-Report der Standish Group erfasst seit 1994 durch Umfragen den Erfolg von IT-Projekten. Im Mittel wird dort die Aussage getroffen, dass ca. $30 \%$ der Projekte erfolgreich sind, 50 \% erfüllen nicht die Erwartungen und $20 \%$, werden abgebrochen oder mit neuem Team und neuer Technologie erneut gestartet. Dabei hat sich diese Verteilung über die vielen Jahre kaum geändert. Auch Ansätze wie das Capability Maturity Modell und die agilen Entwicklungsmethoden haben zu keiner signifikanten Steigerung des Erfolgs geführt.

Eine mögliche Erklärung dieses Phänomens kann die Studie „Benefits of plm“ liefern, die zwischen 2004 und 2009 in drei Stufen durchgeführt wurde. Abb. 1 zeigt, dass mit den Themen Prozesse und Organisation 50 \% der Erfolgsfaktoren humanzentriert sind, während die klassische IT nur einen Beitrag von $10 \%$ am Erfolg liefert. 
Die Studie adressiert auch die notwendige Durchgängigkeit der Unternehmensstrategie, der daraus abgeleiteten Domänenstrategie und den daraus resultierenden Prozess- und IT-Initiativen.

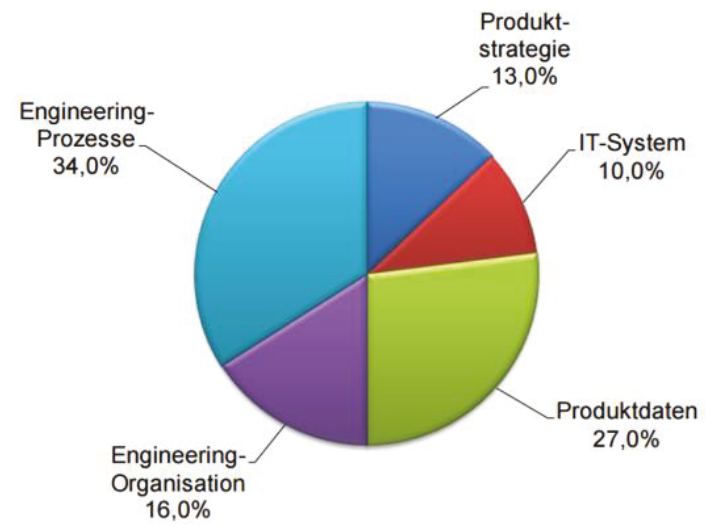

Abbildung 1: Beitragsleister zum plm-Erfolg — das IT-System hat den geringsten Einfluss

IT-Projekte oder die Einführung neuer Technologien sind kein Selbstzweck, sondern sind in der Regel aus dem Bedarf an Optimierung im Sinne der Effizienz und Effektivität abgeleitet. Veränderungsbedarf kann auch durch sich verändernde Wettbewerbssituationen

oder gesetzliche Anforderungen entstehen. Mit der Einführung neuer Systeme verändern sich Geschäftsprozesse und Arbeitsmethoden. Dies funktioniert nur dann erfolgreich, wenn sich auch die handelnden Personen in ihren Handlungsmustern, in der Zusammenarbeit und ihrem Wertschöpfungsbeitrag mit verändern. Daraus lässt sich eindeutig die These ableiten:

Wer Prozess-IT-Projekte als Organisations- und Veränderungsprojekte betrachtet und entsprechend bearbeitet, wird eine deutlich höhere Erfolgswahrscheinlichkeit haben.

Der von Simon Sinek entwickelte "Golden Circle“ liefert eine Erklärung, warum manche Unternehmen deutlich erfolgreicher sind als andere, die zum Teil viel bessere Randbedingungen haben. Dies kann direkt auf IT-Projekte übertragen werden. Die Projekte müssen ein Ziel verfolgen, das von allen beteiligten Akteuren positiv emotional aufgeladen ist, das als attraktiv angesehen wird. Die Frage nach dem „Warum“ muss eindeutig beantwortet werden und die Zielerreichung erstrebenswert sein. Es muss gewollt sein. Nicht nur von dem IT-Leiter und seinen Mitarbeitern, sondern vor allem von dem 
oberen Management. Wenn die Unternehmensleitung eine sinnstiftende und plausible Erklärung hat, warum dieses Projekt für die Zukunft des Unternehmens wichtig ist und dies in angemessener Form kommuniziert, dann ist ein wichtiger und großer Schritt in Richtung Erfolg getan.

Die Arbeiten von Geoffrey Moore, "Crossing the Chasm“, und Gunter Dueck , Das Neue und seine Feinde", klassifizieren vier Verhaltensmuster von Menschen in Organisationen. Während Moore sich auf den Übergang von den Innovators und Early Adopters zu der Early Majority betrachtet, widmet sich Dueck auch stark dem Übergang von der Early zur Late Majority.

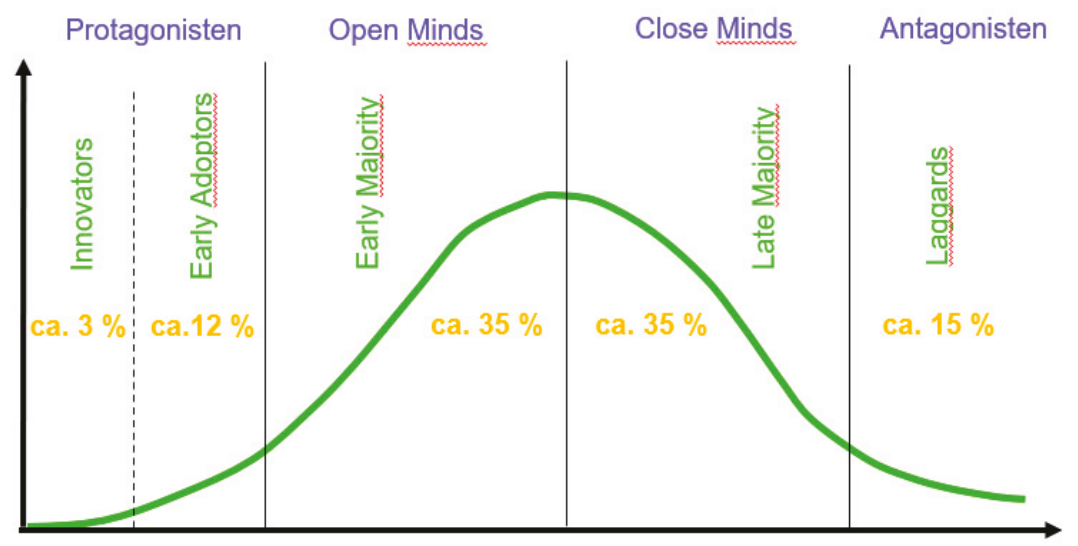

Begriffe nach Dueck, G. „Das Neue und seine Feinde ", 2015

Begriffe nach Moore, G. ${ }_{n}$ Crossing the Chasm ${ }^{\mu}, 2014$

Abbildung 2: Mindset in Veränderungsprojekten nach G. Moore und G. Dueck

Um die Menschen in einem solchen Projekt angemessen „mitzunehmen“, Betroffene zu Beteiligten zu machen und eine positive Veränderungsenergie zu erzeugen, bedarf es verschiedener Phasen, die zu durchlaufen sind. Diese Phasen korrespondieren in einem hohen Maß mit den Verhaltensmustern der unterschiedlichen Personengruppen in Abb. 2. Es ist dabei wichtig zu betonen, dass für ein erfolgreiches Projektergebnis die Mitarbeit von Menschen aus allen vier Gruppen notwendig und zielführend ist. Jede Gruppe beschäftigt sich mit anderen Fragestellungen und Denkweisen und jede leistet einen wichtigen Beitrag zum Projekterfolg. 


\section{Das Fünf-Phasen-Modell}

Das vorgestellte „Fünf-Phasen-Modell“ ist das Ergebnis langjähriger Tätigkeit und Verantwortung für solche IT-Veränderungsprojekte, die nicht immer so gradlinig verlaufen sind, wie man es sich erwartet hat. Häufig haben sich im Projektverlauf der Fokus und die Zielsetzung fundamental geändert, die Projekte sind dann aber im Sinne des Unternehmens positiv, wenn auch mit Verzögerungen und Budgetüberschreitungen, beendet worden. Andere Projekte wurden aber auch ergebnislos abgebrochen. Besonders die Projekte sind gefährdet, bei denen eine große Anzahl von betroffenen Prozessen und Rollen miteinander integrativ behandelt werden sollen.

Die konstruktiv kritische Betrachtung der Erfolge vieler durchgeführter Projekte hat dieses Modell entstehen lassen. Dabei ist ein Schwerpunkt der dritte Übergang von der Late Majority zu den Laggards, dem bisher in der Literatur nur wenig Bedeutung zugemessen wurde, der aber für die Nachhaltigkeit des Projekterfolgs besonders wichtig ist.

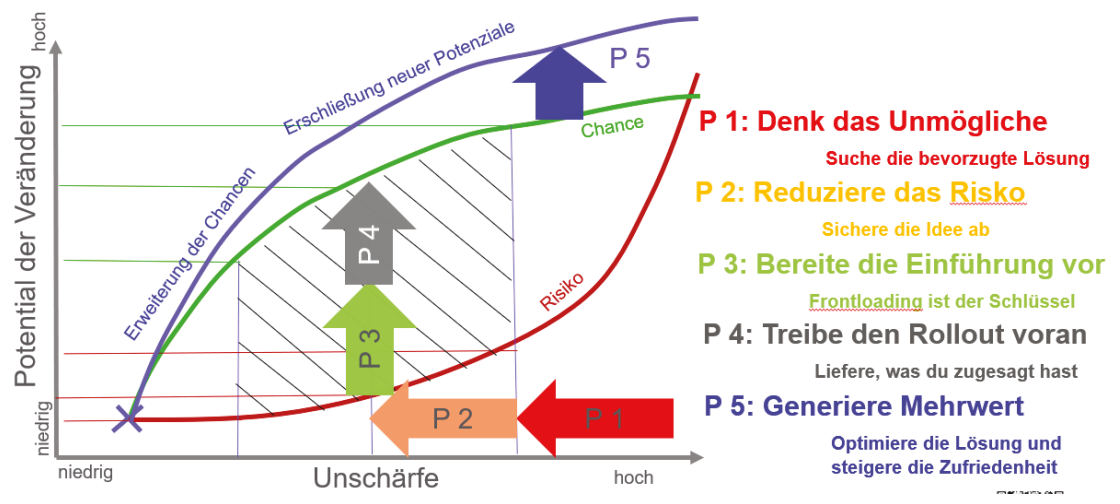

Abbildung 3: Feld der Veränderung

Der Grundgedanke beruht auf der Annahme, dass sich aus dem Status quo, der Komfortzone, bei geringer Unschärfe deutliche Chancen erarbeiten lassen, ohne dabei ein großes Risiko einzugehen. Bei steigender Unschärfe steigt das Risiko progressiv an, während sich der Chancenzuwachs degressiv verhält. Dadurch ergibt sich ein Bereich, das „Feld der Veränderung", in dem das Verhältnis von Chancen zu Risiken besonders groß ist.

Die Phase 1 und 2 des Vorgehensmodells adressieren die Reduktion der Unschärfe. Die Phase 3 und 4 erschließen die Chancen. Phase 5 dient im Sinne von Lessons learnt 
und kontinuierlicher Verbesserung der Erweiterung der Chancen und Erschließung weiterer Potenziale.

\section{Phase 1: „Denke das Unmögliche“ - Suche die bevorzugte Lösung}

Zu einem gewissen Zeitpunkt ist die Notwendigkeit einer Veränderung oder eines Projekts möglicherweise für viele Beteiligte und Betroffene noch gar nicht erkennbar. Auf der anderen Seite herrschen sehr verschwommene Vorstellungen über mögliche Potenziale, Perspektiven und Optionen. Gleichzeitig sind die Erwartungen der einzelnen Stakeholder sehr divergent und die unterschiedlichen Sichtweisen werden aus Konfliktvermeidungsgründen nicht ausdiskutiert.

Diese Phase erfordert die Innovatoren, deren Aufgabe es ist, alle möglichen (und auch die unmöglich erscheinenden) Optionen aufzuzeigen und mit klaren Kriterien zu klassifizieren. Es ist dabei auch sinnvoll und hilfreich, Grenzwertbetrachtungen vorzunehmen und bewusst alles in Frage zu stellen, was bisher handlungsleitend war. Die Auswahlkriterien müssen klar und transparent sein. Dabei gilt es, die verschiedenen Stakeholder mit spezifischen, für sie wichtigen, Kriterien zu berücksichtigen. Während die späteren Anwender der Systeme sich schwerpunktmäßig mit der Funktionalität und der Bedienbarkeit beschäftigen, interessiert das operative Management mehr die Frage der Partner-Interoperabilität und der möglichen Migration. Das obere Management erwartet Antworten zur strategischen Einbindung in die Unternehmenslandschaft und zum Business-Case.

Hilfreich ist auch diese Projektphase in die Hände einer verantwortlichen Person zu geben, die über sehr gute analytische Fähigkeiten verfügt, aber in dem adressierten Themengebiet keine Vergangenheit hat. Dies sichert die Neutralität und Unabhängigkeit in der Auswahl. Das Projektteam kann in dieser Phase aus einer überschaubaren Anzahl von Spezialisten bestehen, die aber ein Höchstmaß an kommunikativen Fähigkeiten mitbringen müssen, um die Gesamtorganisation an ihren Arbeiten und Ergebnissen teilhaben zu lassen. Die Auswahl kann dabei auch durch einen PoC (Prove of Concept) abgesichert und plausibilisiert werden. Am Ende dieser Phase gibt es eine Richtungsempfehlung für eine (oder zwei) Lösung(en), die dann in der Phase 2 zu einer Entscheidungsempfehlung weiter ausgearbeitet werden.

\section{Phase 2: „Reduziere das Risiko“ - Sichere die Idee ab}

In dieser Phase, der Phase der „Early Adopter“, gilt es nun, Betroffene zu Beteiligten zu machen und anhand von repräsentativen Geschäftsszenarien im Detail die Stärken und Schwächen der ausgewählten Lösung zu bewerten und die Potenziale aufzuzeigen. 
Die Szenarien sollten die richtige Balance zwischen Vollständigkeit und Aufwand haben. Es muss sichergestellt sein, dass alle wesentlichen betroffenen Geschäftsprozesse betrachtet werden, aber auf der anderen Seite soll das Ergebnis in einer angemessenen Zeit mit einem vertretbaren Aufwand erzielt werden. Hierbei ist auf eine transparente und faire Bewertung jedes einzelnen Geschäftsprozesses zu achten. In die Untersuchung sind auch Schlüsselanwender mit einzubinden. Die einzelnen Ergebnisse müssen von den betroffenen Verantwortlichen für die jeweiligen Geschäftsprozesse geprüft und abgenommen werden.

Anhand dieser repräsentativen Prozesse ist es nun möglich, Fragen zu den Anwendungsmethoden, zur Einbindung in die internen Geschäftsprozesse bzw. der externen Partnerintegration, zur Datenhaltung, Migration von Altdaten und ähnliches zu betrachten. Auch können in Phase 2 erste Aussagen über den zu erwarteten ChangeProzess, die notwendige Mitarbeiterqualifizierung und die Auswirkungen auf die ITInfrastruktur erarbeitet werden.

In dieser Phase sollte die Projektleitung eine breite Akzeptanz bei den Stakeholdern und Betroffenen haben. Dabei spielt die Balance des Erfahrungshintergrundes zwischen Anwendung und IT eine genauso große Rolle, wie die Planungsfähigkeit bezüglich Zeit, Kosten und Qualität für die spätere Umsetzung.

Die Phase endet mit einer klaren Entscheidung der Geschäftsleitung mit Umsetzungsund Businessplan.

\section{Phase 3: „Bereite die Einführung vor“ - Frontloading ist der Schlüssel}

Nun gilt es, den Rollout vorzubereiten. Während man sich in Phase 2 auf die wichtigsten repräsentativen Geschäftsprozesse konzentrieren konnte, liegt der Fokus nun auf der Vollständigkeit der Prozessabdeckung. Jeder Geschäftsprozess sollte in der neuen Systemwelt an einem oder auch mehreren Beispielen mit realen Daten abgesichert und dokumentiert werden.

Ab dieser Phase ist es sinnvoll, die Projektverantwortung an eine Person zu übertragen, die die Geschäftsprozesse gut kennt und ein hohes Maß an Akzeptanz bei den unterschiedlichen Projektbeteiligten genießt.

Alle Ergebnisse und Annahmen aus Phase 2 müssen nun abgesichert werden. Nötige Nachbesserungen an dem Customizing der Systeme, den Schnittstellen usw. werden dokumentiert, bewertet und eingearbeitet. In dieser Phase können die Akzeptanz und die Zusammenarbeit zwischen den Anwendern und den IT-Verantwortlichen maßgeblich gesteigert werden. Hier gilt es, offene Fragestellungen und Probleme schnell zu 
klären und zu lösen. Das direkte Feedback zu erarbeiteten Problemlösungen ist auch eine mentale Entwicklung der Umsetzungsorganisation zur Vorbereitung auf den Rollout. Auch eine akribische, engagierte und umfassende Vorbereitung in Phase 3 wird nicht verhindern können, dass es im Rollout, in Phase 4, größere und schwerwiegende Probleme geben wird. Wenn sich hier ein gut eingespieltes Team mit einem großen gegenseitigen Verständnis und Vertrauen dieser Probleme annimmt und sie bearbeitet, dann ist die Erfolgswahrscheinlichkeit sehr hoch.

Neben der Phase 2 können auch in Phase 3 mit agilen Entwicklungsmethoden besonders schnell belastbare und akzeptierte Ergebnisse erzielt werden. Dies kann aber nur gelingen, wenn die notwendigen Voraussetzungen für ein agiles Arbeiten auch bei allen Beteiligten geschaffen und gelebt werden.

Die Durchführbarkeit der Geschäftsprozesse muss an Beispielen vollständig beschrieben und die bei der Projektgenehmigung prognostizieren Effekte verifiziert und validiert werden. Aus der Dokumentation werden die Trainingsinhalte abgeleitet. Es kann auch hilfreich sein, die jeweiligen Prozesse in Videos zu dokumentieren, die dann als Hilfe-Funktion für die späteren Anwender zur Verfügung gestellt werden. Am Ende von Phase 3 sind der Rollout-Plan und das dazugehörige Trainingskonzept, inkl. ggf. nötiger Datenmigrationspläne, erarbeitet und mit allen Beteiligten vereinbart. Gleiches gilt bezüglich des Zusammenspiels mit den externen Partnern (Kunden, Lieferanten, Dienstleistern).

\section{Phase 4: „Treibe den Rollout voran“ - Liefere, was du zugesagt hast}

Phase 4 adressiert die Mehrheit der Anwender, die Early und die Late Majority. Während in den bisherigen Phasen agile Entwicklungsmethoden und Vorgehensmodelle sehr sinnvoll und zielführend sind, darf in der Rollout-Phase bei den Anwendern wie auch im Management kein Zweifel an der Ernsthaftigkeit der Durchführung des vorher vereinbarten Plans bestehen. Natürlich werden in der konkreten Umsetzung noch Probleme auftauchen, die bearbeitet werden müssen und die möglicherweise auch zu Konflikten führen können. Deshalb ist es sinnvoll, von vorne herein eine Task-Force einzurichten, die alle aufkommenden Themen aufgreift, klassifiziert, bewertet und, entsprechend des vorher festgelegten Prioritäts-Schemas, abarbeitet. Wenn dieses Team bereits in der Phase 3 gut eingespielt wurde, kann das hier nur hilfreich sein. Hierbei ist es wichtig, sehr stringent und analytisch vorzugehen und die wichtigen Dinge richtig zu tun, anstatt in operative Hektik zu verfallen und die Themen zu bearbeiten, nach denen am lautesten geschrien wird. Hier kann die Analogie zur Medizin weiterhelfen. Ein Arzt macht eine Anamnese, er lässt sich die Symptome beschreiben, 
dann erstellt er eine Diagnose, er ermittelt die wahrscheinlichste Ursache und empfiehlt eine Therapie. Sollte diese nicht die erwartete Wirkung haben, beginnt der Prozess von vorn.

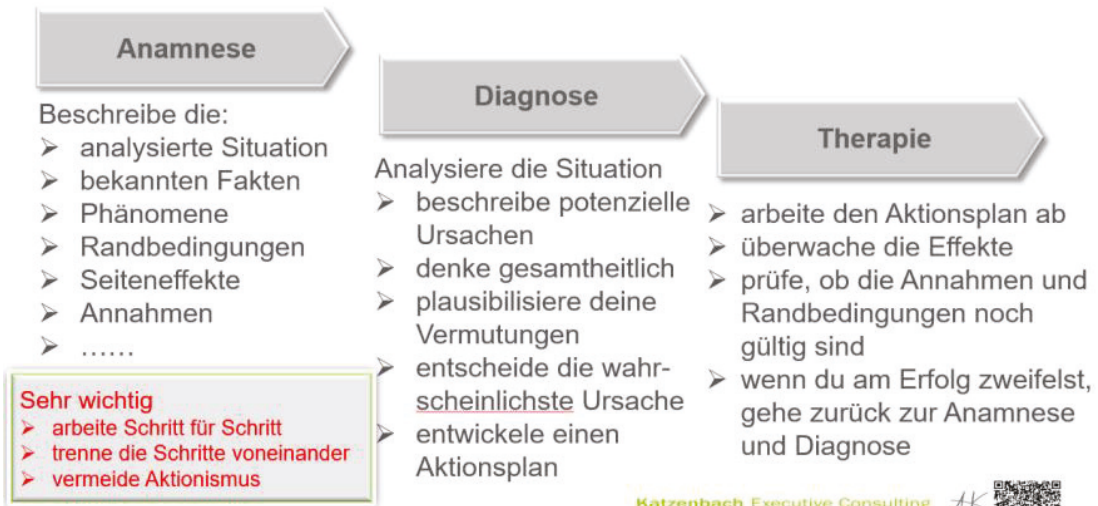

Abbildung 4: Vorgehensmodell des Problem-Managements

Dabei ist es wichtig, dass es bei der Beschreibung des Themas, resp. Problems, keine unterschiedlichen Sichten geben darf. Nur wenn bezüglich der Anamnese Konsens besteht, kann eine qualifizierte Diagnose erstellt und die richtigen Maßnahmen effektiv eingeleitet werden. Hierzu ein provokanter Erfahrungswert: die Ursache der genannten Probleme sind in der Regel nur zu einem kleinen Teil in der auszurollenden Software zu finden (in Analogie der Studie Benefits of plm).

Eine große Bedeutung hat in dieser Phase auch die Priorisierung der Maßnahmen. Hier kann viel Zeit und Geld verschwendet werden, da häufig Ressourcen denjenigen zugeordnet werden, die es besonders gut verstehen, ihren Argumenten öffentlichkeitswirksam Nachdruck zu verleihen. Oft sind aber die Themen der ruhigen Analytiker, die sich weniger kommunikationsintensiv äußern, aber die wichtigeren und entscheidenden. Methodische Hilfestellung gibt der "Leitfaden zur Fehlerklassifizierung für Software", die vom BITKOM 2007 veröffentlicht wurde. Dabei sollte zwischen der Klassifizierung des Themas im Sinne einer Beeinträchtigung der Systemnutzung und einer Priorisierung im Sinne des Einflusses des Themas auf die Geschäftsprozesse unterschieden werden. Aus der Kombination von Klassifizierung und Priorisierung können die Themen identifiziert werden, die vordringlich bearbeitet werden müssen. 


\section{Phase 5: „Generiere weiteren Mehrwert“ - Optimiere die Lösung und steigere die Zufriedenheit}

Nach der Phase 4 werden die meisten Projekte abgeschlossen und „an die Linie übergeben“. Damit verschenkt man sich einen sehr großen Teil des möglichen Potenzials. Jetzt kommt die Zeit der Laggards, die wenig veränderungsbereit sind und "schon immer wussten, dass das alles nicht funktioniert". Oft sind das Menschen, die sich über eine lange Beschäftigungsdauer und gute Arbeit für das Unternehmen in der Vergangenheit verdient gemacht haben und beim oberen Management bekannt sind und dort Vertrauen genießen. Wenn es gelingt, diese Menschen konstruktiv und ergebnisorientiert einzubinden, kann dies einen weiteren Mehrwert darstellen. Wenn diese Vertrauensposition aber missbraucht wird, kann selbst ein objektiv gut gelaufenes Projekt noch in ein schlechtes Licht gerückt und falsch wahrgenommen werden.

Aus der Phase 4 sind auch sicher noch eine Menge offener Probleme und Ideen übriggeblieben, die jetzt im Sinne einer weiteren Optimierung noch einmal bewertet werden sollten. Gleichzeitig kommen durch die Breite der Anwenderschaft neue Themen auf, die ebenfalls eine Betrachtung wert sind. Deshalb sollte man sich für diese Phase durchaus etwas längere Zeit nehmen und auch von vorne herein dafür Ressourcen einplanen und vorsehen.

Das "medizinische“ Vorgehensmodell und die Klassifizierungsmethode analog Phase 4, sollte dabei aber Bestand haben und konsequent angewendet werden.

\section{Zusammenfassung}

Auch wenn sich dieses fünfstufige Prozessmodell für ein erfolgreiches IT-Einführungsprojekt sehr einfach und logisch anhört, so wird es doch im Detail immer wieder Herausforderungen und Schwierigkeiten geben. Hier liegt die Herausforderung darin, den teilweise emotionalen und von zwischenmenschlichen Konflikten geprägten Handlungsmuster durch ein klares und transparentes Vorgehensmodell zu begegnen. Es gilt dabei, die richtige Balance zwischen Kontinuität und Stringenz im Vorgehensmodell auf der einen Seite und der Flexibilität und situativen Anpassungsfähigkeit auf der anderen Seite zu finden.

Grundvoraussetzung ist eine klare, vom Top-Management getragene Zielsetzung, die sich an der strategischen Weiterentwicklung des Unternehmens orientiert. Dabei muss aufgezeigt werden, welchen Beitrag das Projekt zur Erreichung dieser leistet und wie dieser sich in qualitativen und quantitativen Kenngrößen messen lässt. 
Wenn diese Grundvoraussetzung erfüllt ist, dann können mit dem aufgezeigten FünfStufen-Plan auch sehr komplexe und große Projekte mit vielen unterschiedlichen Beteiligten erfolgreich „in time“, „in budget" und „in quality“ durchgeführt werden. Doch die Methodik ist nicht alles, denn es braucht auf jeden Fall

- ein realistisches und skalierbares Umsetzungskonzept,

- hochengagierte und motivierte Mitarbeiter und Partner,

- die zusammenarbeitsfähig sind und gemeinsam im Sinne des "Warums" etwas erreichen wollen,

- eine "Never Give-Up"-Mentalität

- und: Kommunikation, Kommunikation und Kommunikation.

\section{Literaturverzeichnis}

Standish Group, (fortlaufend), Chaos Report: www.standishgroup.com.

Abramovici, M. ad all, 2009, Benefits of plm, Universität Bochum in Zusammenarbeit mit IBM.

Sinek, S. (2011), Start with Why, ISBN: 978-1-59184-644-4.

Moore, G (2014), Crossing the Chasm, ISBN 978-0-06229-300-8.

Dueck, G. (2013), Das Neue und seine Feinde, ISBN 978-3-593-39717-7.

Wolf, G., Leszak, M., (2007), Fehlerklassifikation für Software - Leitfaden, BITKOM.

\section{Kontakt}

Prof. Alfred Katzenbach

Katzenbach Executive Consulting Lehrbeauftragter an der Uni Stuttgart, Institut für Konstruktionslehre und Technisches Design, IKTD Himmernstr. 18 78343 Gaienhofen www.katzenbach-web.de 